\title{
Efficacy of a standardized Centella asiatica (ECa 233) extract against allodynia in a mouse model of temporomandibular osteoarthritis
}

\author{
Nattapon Rotpenpian ${ }^{\mathrm{a}, \mathrm{b}}$, Aree Wanasuntronwong ${ }^{\mathrm{c}}$, Sompol Tapechum ${ }^{\mathrm{a}}$, Chit Care ${ }^{\mathrm{a}}$, Atitaya Roumwong ${ }^{\mathrm{d}}$ \\ Kanokwan Tilokskulchai ${ }^{\mathrm{a}}$, Mayuree H. Tantisira ${ }^{\mathrm{e}}$, Narawut Pakaprot ${ }^{\mathrm{a}, *}$ \\ a Department of Physiology, Faculty of Medicine Siriraj Hospital, Mahidol University, Bangkok 10700 Thailand \\ b Department of Oral Biology and Occlusion, Faculty of Dentistry, Prince of Songkla University, Songkhla 90110 \\ Thailand \\ c Department of Oral Biology, Faculty of Dentistry, Mahidol University, Bangkok 10400 Thailand \\ d Department of Anatomy, Faculty of Medicine, Chulalongkorn University, Bangkok 10330 Thailand \\ e Faculty of Pharmaceutical Sciences, Burapha University, Chonburi 20131 Thailand
}

*Corresponding author, e-mail: narawut.pak@mahidol.ac.th

ABSTRACT: Prolonged inflammation causing tissue injury can induce chronic pain hypersensitivity (allodynia and hyperalgesia). Osteoarthritis of the temporomandibular joint (TMJ-OA) is a common cause of chronic allodynia encountered in dental practice, but many currently available treatments induce intolerable side effects. In this study, we investigated the potential efficacy of a standardized Centella asiatica extract (ECa 233) on allodynia in a TMJ-OA mouse model established by an injection of complete Freund's adjuvant (CFA) into the TMJ. After CFA injection, animals daily received oral administration of vehicle, $0.14 \mathrm{~g} / \mathrm{kg}$ ibuprofen (positive control), $30 \mathrm{mg} / \mathrm{kg}$ ECa 233, or $100 \mathrm{mg} / \mathrm{kg}$ ECa 233. Behavioral pain responses were examined by air-puff tests before and after CFA injection on days 3, 7, 14, 21, and 28. On day 28, TMJ-OA pathology was assessed by changes in articular cartilage thickness and graded according to the Osteoarthritis Cartilage Histopathology Assessment (OCHA) system. In the CFA + vehicle group, pain response scores increased gradually, reaching statistical significance compared to untreated Sham controls on days 14, 21, and 28. On day 28, OCHA grade score was $3.5 \pm 0.35$, and articular cartilage thickness was reduced compared to the Sham group. Both ECa 233 doses significantly attenuated pain response scores and also slowed degeneration of the TMJ with efficacy comparable to ibuprofen. We conclude that ECa 233 can protect against mechanical allodynia and cartilage degeneration in a mouse model of TMJ-OA.

KEYWORDS: temporomandibular joint osteoarthritis, chronic pain, complete Freund's adjuvant, Centella asiatica

\section{INTRODUCTION}

Temporomandibular joint osteoarthritis (TMJ-OA) is a progressive degenerative joint disease characterized by inflammation, destruction of articular cartilage, afferent nerve sensitization, and ensuing chronic inflammatory pain [1]. It is the most common cause of chronic TMJ pain and can occur at any age but is especially frequent in dental patients older than 40 years $[2,3]$. The primary symptom is pain at the TMJ, and surrounding areas evoked by normally innocuous stimuli such as air flow, defined as mechanical allodynia $[4,5]$. Nonsteroidal anti-inflammatory drugs (NSAIDs) can effectively relieve the pain and minimize inflammation associated with TMJ-OA, but long-term use may have adverse effects including peptic ulcer and increased risks for kidney and cardiovascular diseases [6]. Further, a substantial number of cases are refractory to NSAIDs, necessitating the introduction of more effective and safer alternatives.

Among potential treatments with well documented efficacy and safety for other conditions are traditional herbal extracts containing concentrates of bioactive phytochemicals. Centella asiatica (L.) Ur- ban (Indian pennywort, Gotu kola or Bua-bok) is a common component of traditional herbal medicines with reported anti-inflammatory and antinociceptive properties [7]. The major bioactive components of Centella asiatica extracts are triterpenoid glycosides including asiaticoside and madecassoside [7]. However, the analgesic efficacy of these preparations is inconsistent [7] likely due to the variation in active ingredient concentrations. To overcome this problem, guidelines have been established for controlling the preparation process, yielding a standardized Centella asiatica extract termed ECa 233 containing not less than $80 \% \mathrm{w} / \mathrm{w}$ triterpenoid glycosides and a madecassoside to asiaticoside ratio of 1.5 $\pm 0.5: 1$ [8].

This ECa 233 preparation has demonstrated a favorable safety profile in both acute and chronic toxicity tests. Mice and rats receiving $10-1000 \mathrm{mg} / \mathrm{kg}$ for 7 days or 90 days by oral administration showed no alterations in hepatocyte histopathology [9]. Further, ECa 233 neither activated nor inhibited hepatic enzymes that can eliminate or detoxify other major analgesic such as paracetamol and NSAIDs [8], suggesting no interference with drug metabolism. Moreover, ECa 233 at 30 and $100 \mathrm{mg} / \mathrm{kg}$ has been shown to reduce 
experimental thermal pain responses in rodents as assessed by the tail flick test [10], while an oral paste containing ECa 233 significantly decreased inflammation and pain in patients with aphthous ulcers [11]. Thus, ECa 233 appears to be a reliable antinociceptive and anti-inflammatory treatment. A few studies have also investigated the antinociceptive efficacy of Centella asiatica extract on knee joint osteoarthritis [12], but ECa 233 has not been examined for effects on pain associated with TMJ-OA.

Osteoarthritis results from the local release of inflammatory factors such as bradykinin, histamine, serotonin, and prostaglandins in response to minor tissue damage, leading to more extensive tissue injury and a self-perpetuating cycle of progressive tissue degeneration and increasing pain severity [13]. We therefore speculated that a TMJ-OA model could be established by local exposure to an exogenous proinflammatory agent such as complete Freund's adjuvant (CFA). In fact, previous studies have demonstrated that CFA injection into the TMJ of mice causes inflammation and induces behavioral signs of chronic TMJ-OA-like pain in response to non-noxious stimuli $[14,15]$ possibly by inducing sustained activation of primary pain afferents. Chronic activation of these afferents can result in peripheral sensitization and ultimately in central pain sensitization (allodynia and hyperalgesia) [16].

The aim of the present study is to examine the potential efficacy of ECa 233 for reducing behavioral pain responses in a TMJ-OA mouse model established by daily unilateral CFA injection. Moreover, we also examined the structural changes of the TMJ induced by CFA injection to confirm the validity of the model. To our best knowledge, this study is the first to demonstrate the possible benefits of Centella asiatica extract ECa 233 on both TMJ-OA pathogenesis and allodynia.

\section{MATERIALS AND METHODS}

\section{Study groups and experimental design}

This study was approved by the Animal Care and Use Committee, Faculty of Medicine, Siriraj Hospital, Mahidol University (SI-ACUP014/2561) and was performed according to the Animal Research: Reporting In Vivo Experiments (ARRIVE) Guidelines. The sample size was calculated to provide $80 \%$ power $(1-\beta)$ with a 95\% confidence interval $(\alpha=0.05)$ [14]. Thirty adult male ICR mice (6 weeks old, 28-32 g initial body weight) were randomly divided into 5 groups of 6 : a Sham treatment group and 4 CFA groups also receiving vehicle, ibuprofen, or one of 2 ECa 233 doses (30 and $100 \mathrm{mg} / \mathrm{kg}$ ). Each group was housed in separate cages under controlled temperature $\left(22 \pm 2{ }^{\circ} \mathrm{C}\right)$, humidity $(45 \% \pm 15 \%)$, and light/dark cycle (12 h/12 h). The experimental design is illustrated in Fig. 1.

\section{Experimental design}

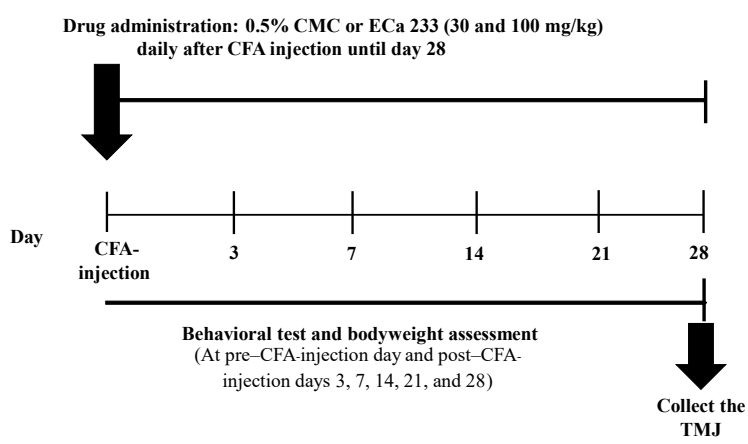

Fig. 1 Experimental design. CFA, complete Freund's adjuvant; CMC, carboxymethyl cellulose; and TMJ, temporomandibular joint (30 and $100 \mathrm{mg} / \mathrm{kg}$ ) daily after CFA injection until day 28 .

\section{Development of the TMJ-OA mouse model}

To induce a condition resembling TMJ-OA, mice were first injected intraperitoneally with sodium pentobarbital $(60 \mathrm{mg} / \mathrm{kg})$ for anesthesia and then with $10 \mu \mathrm{l}$ of pure normal saline (NSS) (Sham group) or NSS containing 50\% CFA (1 mg/ml) (F5881; Sigma-Aldrich, St. Louis, MO, USA) into the right TMJ. Injections were conducted once at the beginning of the experiment according to the previous studies $[14,17,18]$. The anatomical landmark for injection was also described in a previous study [17]. Briefly, to identify the TMJ by palpation, the local hairs were trimmed with a pair of scissors, and a 30-gage needle was inserted through the facial skin until the tip reached the zygomatic arch. The needle was then moved slowly until it passed under the edge of the arch and entered the TMJ space. After the needle reached the TMJ space, $10 \mu \mathrm{l}$ of NSS with or without 50\% CFA was injected slowly over a period of $5 \mathrm{~s}$ under control of a Hamilton syringe $[14,17]$. The body weight of each mouse was monitored throughout the 28-day experimental period to determine whether TMJ pain alters eating habits or general health.

\section{Drug administration and ECa 233 preparation}

After the CFA injection at the beginning, mice in the Sham and CFA + vehicle groups daily received $0.3 \mathrm{ml}$ of $0.5 \%$ carboxymethyl cellulose (CMC) by oral administration. The other $3 \mathrm{CFA}$ groups also daily received an equal volume of CMC containing either (i) $0.14 \mathrm{~g} / \mathrm{kg}$ ibuprofen, (ii) $30 \mathrm{mg} / \mathrm{kg}$ ECa 233, or (iii) $100 \mathrm{mg} / \mathrm{kg}$ ECa 233. The ECa 233 was obtained from Siam Herbal Innovation (Lot No. MRA0511401; Bangkok, Thailand), and ECa 233 suspensions in CMC were prepared daily. All injections and oral administrations 
were conducted at around 8 am.

\section{Behavioral tests for pain sensitivity}

Pain sensitivity was evaluated by applying a 10-psi air puff controlled by a pneumatic pump. Animals were treated in a box container as described in the previous studies $[14,15,19]$. The 10-psi air puff was chosen because it was shown to elicit substantial mechanical allodynia in CFA-injected TMJ-OA mice [15], while a stronger air puff ( $>10$ psi) was inherently noxious and so by definition cannot measure allodynia [14, 15, 19]. Tests were administered both before and after CFA injections on days $3,7,14,21$, and 28 . The air puff was delivered to the right whisker pad (ipsilateral to CFA injection) as described in a previous study [14]. The 28-day measurement period was chosen to assess pain hypersensitivity during both the acute and chronic phases (more than 14 days in animal models according to [20]). We tested 12 times and then recorded response scores as follows: no behavioral response $=$ 0 , head withdrawal $=0.25$, face grooming once $=1$, and face grooming of $>3$ times $=1.5[14,19]$. Response scores were added to obtain the total ipsilateral response scores (maximum of 18).

\section{Structural changes of the TMJ}

After day 28, all mice were deeply anesthetized and transcardially perfused with $250 \mathrm{ml}$ ice-cold phosphate-buffered saline (PBS; $0.1 \mathrm{M}, \mathrm{pH} 7.4$ ). The head was removed and immersed in 4\% paraformaldehyde in PBS (0.1 M, pH 7.4), followed by removal of the skin and opening of the cervical bone. The right TMJ was then excised and decalcified in 10\% formic acid for 7 days before paraffin embedding, sectioning, and histological staining. The TMJ structures including the condylar head, articular disk, and temporal bone were sagittally sectioned at $10-\mu \mathrm{m}$ thickness using a microtome (Global Medical Instrumentation Inc., Ramsey, MN, USA). Sections were deparaffinized, stained with hematoxylin and eosin (HE, SC-396330; Santa Cruz Biotech, Dallas, TX, USA) diluted 1:100 in PBS, and examined under light microscopy (Carl Zeiss Microimaging $\mathrm{GmbH}$, AxioVision 40 version 4.8.2.0, Germany). Sections were collected at intervals of approximately 10 (10th, 20th, 30th, 40th, 50th, and 60th sections) to measure the articular cartilage thickness and grade the degree of TMJ injury by the Osteoarthritis Cartilage Histopathology Assessment (OCHA) system $[21,22]$. Grading was conducted independently by 3 observers using the following scoring criteria $[14,23]$ : surface intact/cartilage morphology intact $=$ 0 , surface intact with abrasion on the superficial layer $=1$, surface discontinuity $=2$, vertical fissures or clefts $=3$, surface erosion $=4$, sclerotic bone within a denuded surface $=5$, and gross deformation $=6$. We measured the articular cartilage thickness in the same slides (10th, 20th, 30th, 40th, 50th, and 60th)

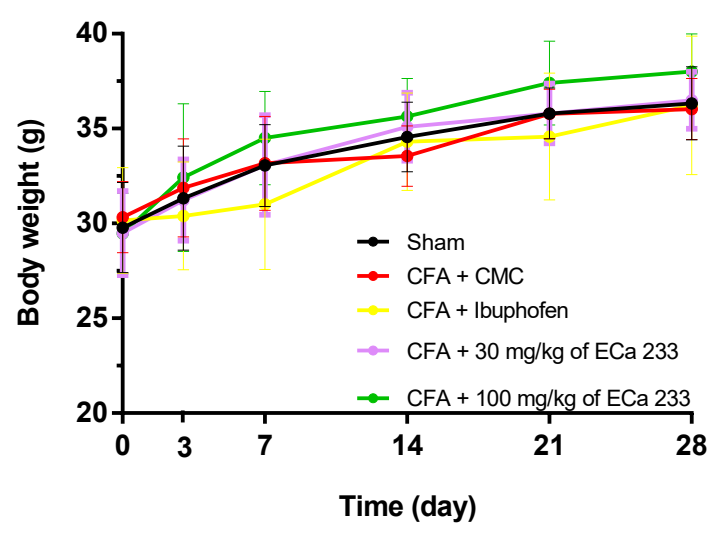

Fig. 2 Effect of CFA-induced temporomandibular joint osteoarthritis (TMJ-OA) on body weight at any time points during the injection protocol. Data are expressed as mean \pm SEM, $n=6$ per group.

examined by the OCHA system. The articular cartilage thickness was measured independently by 3 observers blinded to group allocation at positions where the mandibular condyle head was $20 \%, 50 \%$, and $80 \%$ of total horizontal thickness using ImageJ (hppts:// imagej.nih.gov) [24].

\section{Statistical analysis}

All data, represented from triplicate experiments, are presented as mean \pm standard error of the mean (SEM). Datasets were first tested for normality using the Kolmogorov-Smirnov test. Group means were then compared by two-way analysis of variance (ANOVA) with post hoc Fisher's least significant difference tests for pair-wise comparisons. A $p<0.05$ (two-tailed) was considered statistically significant for all tests. All statistical calculations were conducted using SPSS version 26.0 (IBM, Chicago, IL, USA).

\section{RESULTS}

\section{Body appearance: Weight}

There were no significant differences in body weight among Sham, CFA + vehicle, CFA + ibuprofen, CFA + $30 \mathrm{mg} / \mathrm{kg}$ ECa 233, and CFA + $100 \mathrm{mg} / \mathrm{kg}$ ECa 233 groups after 28 days of treatment (Fig. 2 and Table 1), indicating that induced pain (below) did not interfere with animal feeding or otherwise impair general health and development.

\section{ECa 233 attenuates mechanical allodynia development in a chronic TMJ-OA model}

Fig. 3 illustrates the time course of behavioral pain responses to the 10-psi air-puff test for mechanical allodynia. As expected, the Sham group showed the lowest pain response scores, and responses remained at baseline throughout the 28-day period. In contrast, the CFA + vehicle group demonstrated a progressive 
Table 1 Changes in weekly body weight ( $\mathrm{g}$ ) among treatment groups.

\begin{tabular}{|c|c|c|c|c|c|c|}
\hline Group & Pre-injected & Day 3 & Day 7 & Day 14 & Day 21 & Day 28 \\
\hline 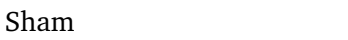 & 78 & 3 & 30 & $34.56 \pm 1.84$ & 4 & 36.3 \\
\hline CFA & 7 & $1.87=$ & $33.17=$ & 33.56 & 35.78 & 36.0 \\
\hline $\mathrm{CFA}+$ ibuprofe & $0.16 \pm 2.77$ & $30.40 \pm 2.84$ & $31.01 \pm 3.43$ & $34.31 \pm 2.56$ & $34.58 \pm 3.34$ & $36.22 \pm 3.85$ \\
\hline $\mathrm{CFA}+30 \mathrm{mg} / \mathrm{kg}$ ECa 233 & $29.49 \pm 2.21$ & $31.23 \pm 2.14$ & $33.08 \pm 2.62$ & $35.10 \pm 1.79$ & $35.80 \pm 1.57$ & $36.49 \pm 1.50$ \\
\hline $\mathrm{CFA}+100 \mathrm{mg} / \mathrm{kg}$ ECa 233 & $29.47 \pm 2.12$ & $32.42 \pm 3.89$ & $34.50 \pm 2.46$ & $35.64 \pm 2.01$ & $37.40 \pm 2.21$ & $38.44 \pm 2.18$ \\
\hline
\end{tabular}

Values presented are mean $\pm \operatorname{SEM}(n=6)$.

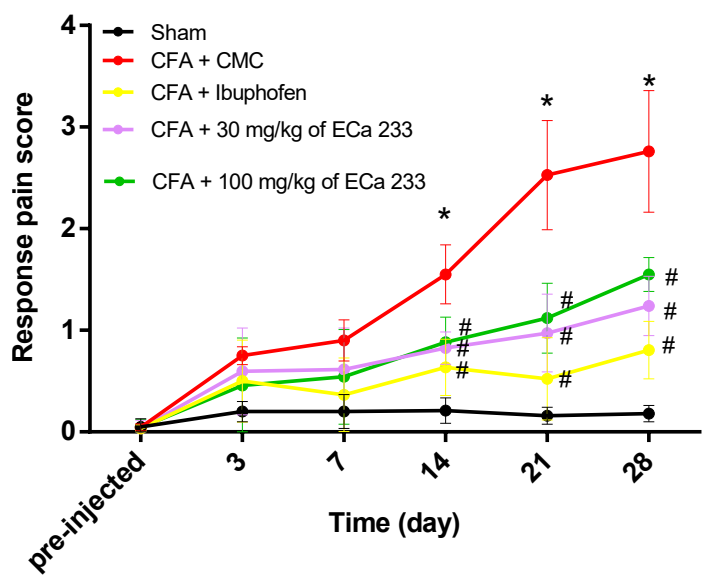

Fig. 3 Effect of ECa 233 on pain response scores at the different time points after CFA-induced temporomandibular joint osteoarthritis (TMJ-OA). Data are expressed as mean \pm SEM, $n=6$ per group. ${ }^{*} p<0.05$ compared to the Sham group. ${ }^{\#} p<0.05$ compared to the CFA + CMC group.

increase in pain response that was significantly higher than in Sham mice on injection days $14[F(4,45)=$ 13.823, $p<0.05], 21[F(4,45)=15.213, p<0.05]$, and $28[F(4,45)=19.149, p<0.05]$. Daily ibupro-

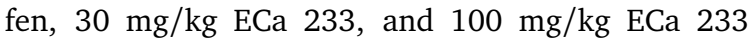
co-treatments all significantly reduced pain responses to air puffs on days 14,21 , and 28 compared to CFA + vehicle group (CFA + Ibuprofen: $[F(4,45)=$ $15.139, p<0.05],[F(4,45)=16.134, p<0.05]$, and $[F(4,45)=17.901, p<0.05] ; \mathrm{CFA}+30 \mathrm{mg} / \mathrm{kg}$ of ECa 233: $[F(4,45)=15.215, p<0.05],[F(4,45)=$ $15.652, p<0.05]$, and $[F(4,45)=14.592, p<0.05]$; $\mathrm{CFA}+100 \mathrm{mg} / \mathrm{kg}$ of ECa 233: $[F(4,45)=18.252, p<$ $0.05],[F(4,45)=16.358, p<0.05]$, and $[F(4,45)=$ $16.014, p<0.05]$, respectively), indicating significant reduction of mechanical allodynia. Further, there were no significant differences in pain response among ibuprofen and ECa 233 groups on any day, suggesting that ECa 233 was roughly as effective as the ubiquitous clinical analgesic.

\section{ECa 233 also alleviates CFA-induced TMJ injuries}

Injuries to the TMJ induced by CFA injection were assessed on histological sections according to the OCHA system. Fig. 4 shows the structural changes of the TMJ condylar head after all treatment protocols. After the CFA injection, the surface of the condylar head had eroded and detached. Mean OCHA system grading score for the right TMJ was $3.5 \pm 0.35$ after 28 days in the CFA + vehicle group but was 0 in the Sham group, ibuprofen group, and both ECa 233 (30 and $100 \mathrm{mg} / \mathrm{kg}$ ) groups. Thus, ECa 233 also prevented TMJ condyle head degeneration as effectively as ibuprofen.

Further, CFA reduced while ECa 233 co-treatment partially preserved normal articular cartilage thickness at positions where the mandibular condyle head is $20 \%, 50 \%$, and $80 \%$ of maximum horizontal thickness. The CFA + vehicle group exhibited a significantly reduced mean articular cartilage thickness on day 28 $[F(4,45)=12.823, p<0.05]$ compared to the Sham group, while articular cartilage thicknesses values did not differ among Sham, ibuprofen, and ECa 233 treatment groups (CFA + Ibuprofen: $[F(4,45)=10.339$, $p=0.253]$, CFA $+30 \mathrm{mg} / \mathrm{kg}$ of ECa $233:[F(4,45)=$ $11.034, p=0.279]$, and CFA $+100 \mathrm{mg} / \mathrm{kg}$ of ECa 233: $[F(4,45)=11.001, p=0.265]$ ) (Fig. 5).

\section{DISCUSSION}

This is the first in vivo study demonstrating that a standardized extract of Centella asiatica (ECa 233) can attenuate pain hypersensitization (mechanical allodynia) and joint degeneration in a CFA injection model of TMJ-OA. During the CFA injection period, food and water intake were not impaired as evidenced by the normal increase in body weight. Stimulation with a normally innocuous air puff induced behavioral signs of pain on days 14,21 , and 28 of CFA + vehicle injection in accord with the previous studies showing that CFA can induce the excessive inflammation and pain hypersensitivity characteristic of TMJ-OA $[19,20]$. Further, we provide additional validation of the model by showing that CFA caused degeneration of the TMJ articular cartilage surface, consistent with previous reports that articular cartilage thickness and mandible bone density were reduced after 21 and 28 days of CFA injection in mice [14, 25].

The pathophysiology of chronic TMJ-OA is still not completely clear but likely involves local release of proinflammatory factors such as bradykinin, histamine, serotonin, and prostaglandins [26]. Similarly, CFA 


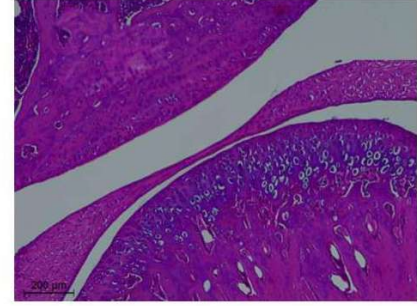

Sham

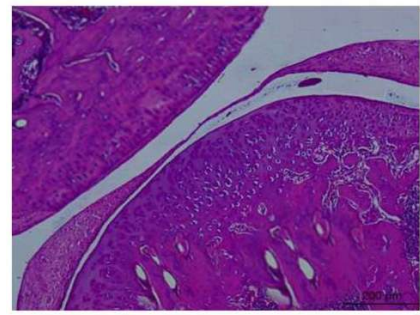

CFA + Ibuprofen

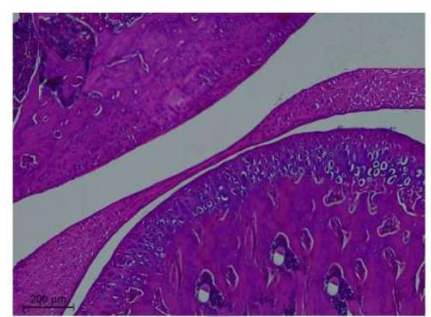

CFA $+30 \mathrm{mg} / \mathrm{kg}$ of ECa 233

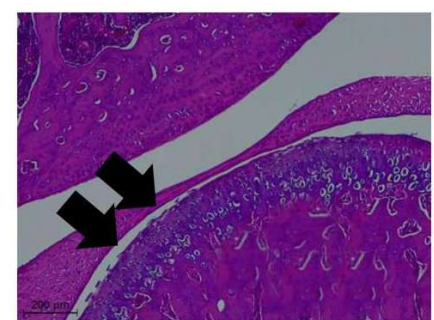

$\mathrm{CFA}+\mathrm{CMC}$

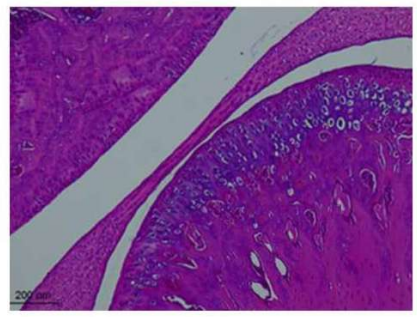

$\mathrm{CFA}+100 \mathrm{mg} / \mathrm{kg}$ of $\mathrm{ECa} 233$

Fig. 4 Effect of ECa 233 on degeneration of the ipsilateral TMJ condyle head articular surface. Representative images of hematoxylin and eosin (HE)-stained sections of the TMJ after 28 days of the CFA injection plus vehicle, ibuprofen, or ECa 233 (scale bar $=200 \mu \mathrm{m}$ ). The black arrows indicate the degeneration of the ipsilateral articular surface.

(a)

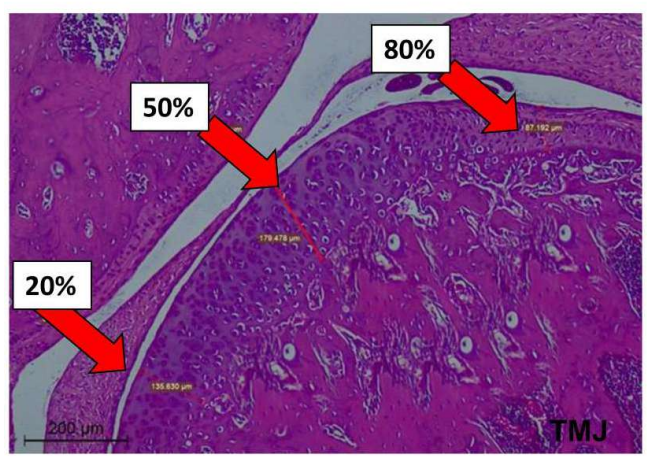

(b)

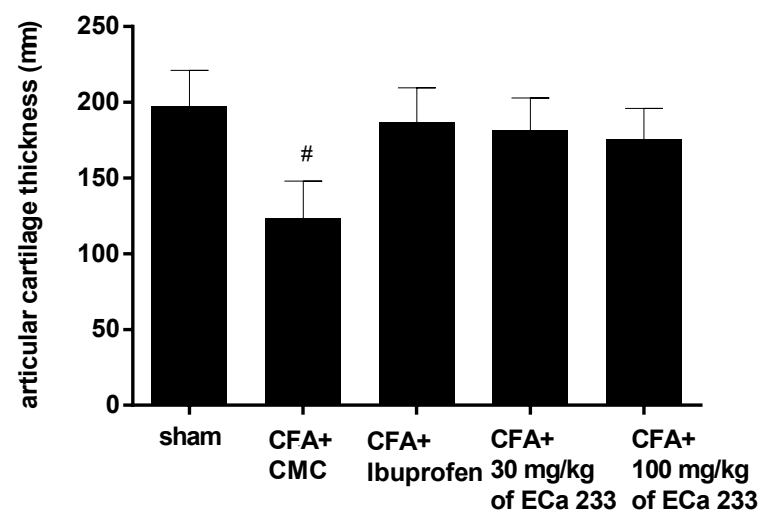

Fig. 5 Effect of ECa 233 on articular cartilage thickness of the condyle head TMJ. (a) Representative measurement of articular cartilage thickness at positions corresponding to $20 \%, 50 \%$, and $80 \%$ of maximum mandible condyle head thickness (red arrows) from hematoxylin and eosin (HE)-stained sections prepared on day 28 of the experimental protocol (scale bar $=$ $200 \mu \mathrm{m}$ ), (b) mean values of articular cartilage thickness in the different groups. Data are expressed as mean \pm SEM, $n=6$ per group. ${ }^{\#} p<0.05$ compared to the Sham group.

injection induces the release of inflammatory mediators that chronically activate primary nerve afferents. Accumulation of these factors and ensuing tissue damage ultimately reduces nerve firing threshold and promotes ectopic synapse formation (plasticity), resulting in increased pain sensitivity at the peripheral level (peripheral sensitization) and eventually at the spinal level (central sensitization) [27]. In allodynia, air flow or light touch can evoke pain sensation possibly by activating low-threshold myelinated A fibers mediating touch sensation that spouts axons and synapse on nociception-transmitting interneurons in laminae I and II of the trigeminal subnucleus caudalis (TNC, which also receives projections from nociceptive $\mathrm{A} \delta$ and $\mathrm{C}$ fibers) [28]. Further study is required to determine if CFA injection induces and ECa 233 reverses myelinated A fiber innervation of the TNC.

The majority of TMJ-OA patients eventually develop abnormalities in TMJ bone structure [26, 27]. These changes may result from chronic inflammation 
as experimentally evoked inflammation in the TMJ resulted in degeneration of the condylar head as well as pain hypersensitivity $[20,29,30]$. Based on these findings, we speculated that CFA would alter TMJ structure via uncontrolled inflammation, thereby mimicking the pathogenesis and clinical features of advanced TMJOA $[20,29]$. Our previous study demonstrated that unilateral CFA injection can induce chronic inflammation in the ipsilateral joint, and allodynia was observed primarily on the ipsilateral side in the current study. Furthermore, this mechanical allodynia was sustained for longer than 14 days, defined as chronic for animal models. We suggest that these long-lasting changes represent central sensitization as Rotpenpian and colleagues reported significantly enhanced phosphorylated cAMP response element-binding protein (pCREB) and microglial activation in the TNC on day 28 of CFA injection [14], indicating long-term hyperactivation of nociceptive pathways.

Both the clinical analgesic ibuprofen and ECa 233 (30 and $100 \mathrm{mg} / \mathrm{kg}$ ) significantly reduced pain response scores compared to vehicle. Moreover, both treatments also protected the TMJ from degeneration as indicated by OCHA system grading scores and articular cartilage thickness measurements of TMJ sections, which are the most reliable assessments of osteoarthritis in animal models [5,31]. Ibuprofen is a drug of choice for pain relief and can protect against osteoarthritis development by blocking COX enzymes and ensuing generation of inflammatory mediators [32-34]. Similarly, the bioactive ingredients in Centella asiatica extracts have demonstrated both antiinflammatory and antinociceptive activities in preclinical studies. For example, like ibuprofen treatment, oral treatment with $100 \mathrm{mg} / \mathrm{kg}$ Centella asiatica extract reduced subsequent carrageenan- and prostaglandininduced hind foot edema and pain in rats [7]. Centella asiatica extract also significantly reduced expression levels of the pro-inflammatory cytokines' tumor necrosis factor- $\alpha$ and interleukin- 6 in lipopolysaccharidetreated HEK-293 cells [35].

The active ingredients of ECa 233 (which contains $\geqslant 80 \% \mathrm{w} / \mathrm{w}$ triterpenoid glycosides and a 1.5:1 ratio of madecassoside to asiaticoside) also possess antiinflammatory and antinociceptive activities when used alone. For instance, oral madecassoside (40 mg/kg) administration reduced the clinical pain scores of mice with induced rheumatoid arthritis of the tail joint and also minimized COX-2 activity and production of prostaglandins, tumor necrosis factor- $\alpha$, and interleukin-6 [35]. Similarly, oral treatment with $10 \mathrm{mg} / \mathrm{kg}$ asiaticoside reduced capsaicin-induced paw pain in mice [36] possibly by increasing release of gamma aminobutyric acid (GABA), the major inhibitory neurotransmitter in the CNS. Indeed, an aqueous extract of Centella asiatica elevated the activity of the GABA synthesis enzyme, glutamic acid decarboxylase (GAD) [37]. Moreover, ECa 233 was reported to activate GABAergic synapses in the amygdala [38]. Increased GABA activity in the CNS may block mechanical allodynia by interfering with the sensitization processes at the neural level. Centella asiatica also reduced pain in an animal model of migraine by blocking the serotonin (5-HT) receptors, 5-HT1A and 5-HT1B [39], and inducing activation of ERK1/2 and Akt signaling pathways [40]. Additional studies are needed to identify the molecular pathways underlying experimental allodynia in this TMJ-OA model and the therapeutic mechanisms of ECa 233 components.

This study has several limitations. First, pain in experimental animals can only be measured by behavioral responses, and behavioral scoring can be highly subjective. Also, we did not examine the progression of articular cartilage damage during the experiment to assess associations with pain responses (on day 3 , 7,14 , and 21 ) as our primary aim was to investigate behavioral and structural changes during the chronic phase (after 28 days of CFA injection). Moreover, we have not yet examined the potential molecular mechanisms underlying these effects of ECa 233 such as reduction of pro-inflammatory cytokine accumulation in the TMJ, suppression of microglial activation in the spinal cord, and downregulation of p-CREB in the TNC.

\section{CONCLUSION}

A standardized Centella asiatica extract (ECa 233) protects against pain hypersensitivity and TMJ cartilage degeneration in a mouse model of chronic TMJ-OA without apparent systemic toxicity. Therefore, clinical trials investigating its efficacy and safety for chronic pain management in OA and other disorders are warranted.

Acknowledgements: This research was supported by a Prince of Songkla University Scholarship, the Thailand Research Fund through the Royal Golden Jubilee PhD program (Grant No. PHD 0058/2561), and Siriraj Research Fund, Faculty of Medicine, Siriraj Hospital, Mahidol University (Grant Number (IO) R016331041). We would like to thank Assist. Prof. Dr. Aunwaya Kaewpitak for helpful suggestions. We would also like to thank research assistants, Anchalee Vattarakorn and Wongsathit Chindasri, for their help with experiments.

\section{REFERENCES}

1. Derwich M, Mitus-Kenig M, Pawlowska E (2020) Interdisciplinary approach to the temporomandibular joint osteoarthritis: Review of the literature. Medicina 56, ID 225.

2. Xu $\mathrm{K}$, Meng Z, Xian XM, Deng $\mathrm{MH}$, Meng QG, Fang W, Zhang D, Long X (2020) LncRNA PVT1 induces chondrocyte apoptosis through upregulation of TNF- $\alpha$ in synoviocytes by sponging miR-211-3p. Mol Cell Probes 52, ID 101560. 
3. de Souza RF, Lovato da Silva CH, Nasser M, Fedorowicz Z, Al-Muharraqi MA (2012) Interventions for the management of temporomandibular joint osteoarthritis. Cochrane Database Syst Rev 2012, ID CD007261.

4. Lee YH, Park HK, Auh QS, Nah H, Lee JS, Moon HJ, Heo DN, Kim IS, et al (2020) Emerging potential of exosomes in regenerative medicine for temporomandibular joint osteoarthritis. Int J Mol Sci 21, 1-21.

5. Abramoff B, Caldera FE (2020) Osteoarthritis: pathology, diagnosis, and treatment options. Med Clin North Am 104, 293-311.

6. Rannou F, Pelletier JP, Martel-Pelletier J (2016) Efficacy and safety of topical NSAIDs in the management of osteoarthritis: evidence from real-life setting trials and surveys. Semin Arthritis Rheum 45, 18-21.

7. Huang SS, Chiu CS, Chen HJ, Hou WC, Sheu MJ, Lin YC, Shie PH, Huang GJ (2011) Antinociceptive activities and the mechanisms of anti-inflammation of asiatic acid in mice. Evid Based Complement Alternat Med 2011, ID 878391.

8. Anukunwithaya T, Tantisira $\mathrm{MH}$, Tantisira B, Khemawoot P (2017) Pharmacokinetics of a standardized extract of Centella asiatica ECa 233 in rats. Planta Med 83, 710-717.

9. Chivapat S, Chavalittumrong P, Tantisira MH (2011) Acute and sub-chronic toxicity studies of a standardized extract of Centella asiatica ECa 233. Thai J Pharm Sci 35, 55-64.

10. Ketkomol P, Towiwat P, Tantisira B, Tantisira M (2012) Anti-nociceptive effects of standardized extract of centella asiatica ECa 233 in tail flick test. Thai $J$ Pharmacol 34, 196-203.

11. Ruengprasertkit C, Hongprasong N, Tantisira B, Tantisira MH (2010) Preliminary study of effects of a standardized extract of Centella asiatica ECa233 on minor aphthous ulcers. CU Dent $J$ 33, 131-142.

12. Micheli L, Di Cesare Mannelli L (2020) Intra-Articular route for the system of molecules 14G1862 from centella asiatica: pain relieving and protective effects in a rat model of osteoarthritis. Nutrients 12, ID 1618.

13. Ouanounou A, Goldberg M, Haas DA (2017) Pharmacotherapy in temporomandibular disorders: a review. $J$ Can Dent Assoc 83, 1-8.

14. Rotpenpian N, Tapechum S, Vattarakorn A, Chindasri W, Care C, Pakaprot N, Wanasuntronwong A (2021) Evolution of mirror-image pain in temporomandibular joint osteoarthritis mouse model. J Appl Oral Sci 29, $1-12$.

15. Krzyzanowska A, Pittolo S, Cabrerizo M, Sanchez-Lopez J, Krishnasamy S, Venero C, Avendano C (2011) Assessing nociceptive sensitivity in mouse models of inflammatory and neuropathic trigeminal pain. $J$ Neurosci Methods 201, 46-54.

16. Bai Q, Liu S, Shu H, Tang Y, George S, Dong T, Schmidt $\mathrm{BL}$, Tao F (2019) TNF $\alpha$ in the trigeminal nociceptive system is critical for temporomandibular joint pain. Mol Neurobiol 56, 278-291.

17. Kim SH, Son CN (2015) Infliximab partially alleviates the bite force reduction in a mouse model of temporomandibular joint pain. J Korean Med Sci 30, 552-558.

18. Morel M, Ruscitto A, Pylawka S, Reeve G, Embree MC (2019) Extracellular matrix turnover and inflammation in chemically-induced TMJ arthritis mouse models. PLoS
One 14, ID 0223244.

19. Krzyzanowska A, Avendaño C (2012) Behavioral testing in rodent models of orofacial neuropathic and inflammatory pain. Brain Behav 2, 678-697.

20. Xu L, Guo H, Li C, Xu J, Fang W, Long X (2016) A timedependent degeneration manner of condyle in rat CFAinduced inflamed TMJ. Am J Transl Res 8, 556-567.

21. Wang D-H, Yang M-C, Hsu W-E, Hsu M-L, Yu L-M (2017) Response of the temporomandibular joint tissue of rats to rheumatoid arthritis induction methods. J Dent Sci 12, 83-90.

22. Artuzi FE, Puricelli E, Baraldi CE, Quevedo AS, Ponzoni D (2020) Reduction of osteoarthritis severity in the temporomandibular joint of rabbits treated with chondroitin sulfate and glucosamine. PLoS One 15, ID 0231734.

23. Pritzker KPH, Gay S, Jimenez SA, Ostergaard K, Pelletier JP, Revell PA, Salter D, van den Berg WB (2006) Osteoarthritis cartilage histopathology: grading and staging. Osteoarthr Cartil 14, 13-29.

24. Nagira K, Ikuta Y, Shinohara M, Sanada Y, Omoto T, Kanaya H, Nakasa T, Ishikawa M, et al (2020) Histological scoring system for subchondral bone changes in murine models of joint aging and osteoarthritis. Sci Rep 10, ID 10077.

25. Lemos GA, da Silva PLP, Batista AUD, Palomari ET (2018) Experimental model of temporomandibular joint arthritis: evaluation of contralateral joint and masticatory muscles. Arch Oral Biol 95, 79-88.

26. Lam DK, Sessle BJ, Cairns BE, Hu JW (2005) Neural mechanisms of temporomandibular joint and masticatory muscle pain: a possible role for peripheral glutamate receptor mechanisms. Pain Res Manag 10, 145-152.

27. Wang XD, Kou XX, Mao JJ, Gan YH, Zhou YH (2012) Sustained inflammation induces degeneration of the temporomandibular joint. J Dent Res 91, 499-505.

28. Lis K, Grygorowicz T, Cudna A, Szymkowski DE, Balkowiec-Iskra E (2017) Inhibition of TNF reduces mechanical orofacial hyperalgesia induced by Complete Freund's Adjuvant by a TRPV1-dependent mechanism in mice. Pharmacol Rep 69, 1380-1385.

29. Chillingworth NL, Donaldson LF (2003) Characterisation of a Freund's complete adjuvant-induced model of chronic arthritis in mice. $J$ Neurosci Methods 128, 45-52.

30. Nicoll SB, Hee CK, Davis MB, Winkelstein BA (2010) A rat model of temporomandibular joint pain with histopathologic modifications. $J$ Orofac Pain 24, 298-304.

31. Aho OM, Finnilä M, Thevenot J, Saarakkala S, Lehenkari P (2017) Subchondral bone histology and grading in osteoarthritis. PLoS One 12, ID 0173726.

32. Mlost J, Kostrzewa M, Malek N, Starowicz K (2018) Molecular understanding of the activation of CB1 and blockade of TRPV1 receptors: implications for novel treatment strategies in osteoarthritis. Int J Mol Sci 19, ID 342 .

33. Kuptniratsaikul V, Dajpratham P, Taechaarpornkul W, Buntragulpoontawee M, Lukkanapichonchut P, Chootip C, Saengsuwan J, Tantayakom K, et al (2014) Efficacy and safety of Curcuma domestica extracts compared with ibuprofen in patients with knee osteoarthritis: a multicenter study. Clin Interv Aging 9, 451-458.

34. Zhang S, Teo KYW, Chuah SJ, Lai RC, Lim SK, Toh 
WS (2019) MSC exosomes alleviate temporomandibular joint osteoarthritis by attenuating inflammation and restoring matrix homeostasis. Biomaterials 200, 35-47.

35. Liu M, Dai Y, Yao X, Li Y, Luo Y, Xia Y, Gong Z (2008) Antirheumatoid arthritic effect of madecassoside on type II collagen-induced arthritis in mice. Int Immunopharmacol 8, 1561-1566.

36. Ayumi RR, Shaik Mossadeq WM, Zakaria ZA, Bakhtiar MT, Kamarudin N, Hisamuddin N, Talib M, Sabar AM (2020) Antinociceptive activity of asiaticoside in mouse models of induced nociception. Planta Med 86, 548-555.

37. Awad R, Levac D, Cybulska P, Merali Z, Trudeau VL, Arnason JT (2007) Effects of traditionally used anxiolytic botanicals on enzymes of the gamma-aminobutyric acid (GABA) system. Can J Physiol Pharmacol 85, 933-942.

38. Wanasuntronwong A, Wanakhachornkrai O, Phong- phanphanee P, Isa T, Tantisira B, Tantisira MH (2018) Modulation of neuronal activity on intercalated neurons of amygdala might underlie anxiolytic activity of a standardized extract of Centella asiatica ECa233. Evid Based Complement Alternat Med 2018, ID 3853147.

39. Bobade V, Bodhankar SL, Aswar U, Vishwaraman M, Thakurdesai P (2015) Prophylactic effects of asiaticoside-based standardized extract of Centella asiatica (L.) urban leaves on experimental migraine: Involvement of 5HT1A/1B receptors. Chin J Nat Med 13, 274-282.

40. Wanakhachornkrai O, Pongrakhananon V, Chunhacha P, Wanasuntronwong A, Vattanajun A, Tantisira B, Chanvorachote P, Tantisira MH (2013) Neuritogenic effect of standardized extract of Centella asiatica ECa233 on human neuroblastoma cells. BMC Complement Altern Med 13, ID 204. 